\title{
Circulating tumor cells and CXCR4 in the prognosis of hepatocellular carcinoma
}

\author{
Tao Bai ${ }^{1,2,3 \#}$, Rongyun Mai ${ }^{1,2,3 \#}$, Jiazhou Ye ${ }^{1,2,3 \#}$, Jie Chen ${ }^{1,2,3 \#}$, Lunan Qi ${ }^{1,2,3}$, Juan Tang ${ }^{1,2,3}$, Meng Wei ${ }^{1,2,3}$, \\ Lianda Zhang ${ }^{1,2,3}$, Zhiwei Chen ${ }^{1,2,3}$, Zhihong Tang ${ }^{1,2,3}$, Lequn $\mathbf{L i}^{1,2,3}$, Feixiang $\mathbf{W u}^{1,2,3}$ \\ ${ }^{1}$ Department of Hepatobiliary Surgery, Guangxi Medical University Cancer Hospital, Nanning 530021, China; ${ }^{2}$ Guangxi Liver Cancer Diagnosis \\ and Treatment Engineering and Technology Research Center, Nanning 530021, China; ${ }^{3}$ Key Laboratory of Early Prevention and Treatment for \\ Regional High Frequency Tumor, Ministry of Education, Nanning 530021, China \\ Contributions: (I) Conception and design: T Bai, R Mai, J Ye, J Chen, L Li, F Wu; (II) Administrative support: F Wu; (III) Provision of study materials \\ or patients: T Bai, R Mai, J Ye, J Chen; (IV) Collection and assembly of data: All authors; (V) Data analysis and interpretation: T Bai, R Mai, J Ye, J \\ Chen; (VI) Manuscript writing: H Sang, S Wu; (VII) Final approval of manuscript: All authors. \\ "These authors contributed equally to this work. \\ Correspondence to: Feixiang Wu, Lequn Li. Department of Hepatobiliary Surgery, Guangxi Medical University Cancer Hospital, He Di Rd. \#71, \\ Nanning 530021, China. Email: wufx2013@163.com; lequn_li001@163.com.
}

Background: This study was to determine circulating tumor cells (CTCs) and the expression of CXC chemokine receptor type 4 (CXCR4) in primary hepatocellular carcinoma (HCC) and the relationships with prognosis.

Methods: We used an advanced CanPatrol ${ }^{\mathrm{TM}}$ CTC-enrichment technique to collect CTCs for isolation and characterization from blood samples. The RNA in situ hybridization (RNA-ISH) method, which is based on branched DNA (bDNA) signal amplification technology, was used to determine the expression of CXCR4 according to epithelial-mesenchymal transition (EMT) markers in 99 patients with primary liver cancer in blood samples pre-operatively. The relationship between the EMT markers and HCC was determined.

Results: The positive rates of CTCs and CXCR4 were $89.9 \%$ and $58.8 \%$, respectively. CTCs were positively correlated with the Barcelona clinic liver cancer (BCLC) staging, tumor diameter and number, envelope, microsatellite damage, portal vein thrombosis, alpha-fetoprotein (AFP), and hepatitis B DNA, and negatively correlated with Edmondson grade. There were significant differences in the expression of CXCR4 between interstitial CTCs and mixed CTCs. A total of 99 patients underwent CTCs testing prior to surgery. The tumor-free survival time of HCC patients with interstitial CTCs <1 (13.3 months) was significantly longer than patients with interstitial CTCs $\geq 1$ (5.0 months) pre-operatively.

Conclusions: CTC-positivity was shown to be associated with HCC and can be used as an independent prognostic factor for HCC. High CXCR4 protein expression was more common in mixed CTCs.

Keywords: Hepatocellular carcinoma (HCC); circulating tumor cells (CTCs); chemokine receptor; prognosis

Submitted Oct 02, 2019. Accepted for publication Dec 26, 2019.

doi: $10.21037 /$ tcr.2020.01.14

View this article at: http://dx.doi.org/10.21037/tcr.2020.01.14 


\section{Introduction}

Hepatocellular carcinoma (HCC) is a major health problem worldwide. there were more than 782,000 cases diagnosed annually, and $50 \%$ in China alone (1). There are currently many treatments for HCC, liver transplantation and surgical resection are the preferred treatment methods (2). But a significant number of patients cannot achieve the expected effect due to post-operative metastasis and recurrence. Microvascular invasion (MVI) can usually be clearly diagnosed in postoperational pathology. And metastases are usually found by chance during resection, both of which are associated with significantly worse prognosis (3). Hence, it is necessary to determine the risk factors for HCC recurrence and the markers for continuous monitoring of anti-tumor response before and after surgery.

Circulating tumor cells (CTCs) is an integral part of "liquid biopsy" and has great potential to change the current treatment modality in the cancer field (4). CTCs are derived from solid tumors and are associated with hematogenous metastasis (5). Therefore, analyzing the level of CTCs has clinical guiding significance. In lung cancer, CTCs exist in dynamic stages of primary tumor detaching and disseminating into the blood (6). For liver cancer patients, overall survival (OS) tended to be poorer in patients with CTCs (7).

The CXC chemokine receptor type 4 (CXCR4) is a specific receptor for the chemokine stromal cell-derived factor-1 (CXCL12). In general, CXCR4 helps activate the immune system and stimulates cell movement, but when the signal that activates the receptor is not properly modulated, CXCR4 accelerates the progression and spread of cancer (8).

CTCs and CXCR4 are indicators associated with prognosis of patients after tumor resection. The relevance of these two indicators has been reported for many tumors, but the relevance between peripheral CTCs and tumor tissue CXCR4 in HCC patients has rarely been reported (9). The relationships between peripheral CTCs, HCC stage, and CXCR4 expression on tumor tissues were analyzed in this study.

\section{Methods}

\section{Patients}

The clinical information of 99 patients with a diagnosis of primary liver cancer that were treated in the Hepatobiliary Surgery Department of our hospital between March 2016 and January 2017 were prospectively analyzed. There were 85 males and 14 females with an age range of 20 72 years, and an average age of $46.19 \pm 10.12$ years (see Table 1 for Baseline data of 99 patients enrolled in CTCs). One hundred thirty detections of CTCs were performed; 99 patients agreed to perform CTCs detection once preoperatively. Statistical analysis was based on pre-operative/ pre-therapy data. The clinical diagnosis standard for the patients was in accordance with the 2015 edition of The Standardized Case Diagnosis Guidelines of Primary Liver Cancer. None of the patients had received targeted therapy, radiotherapy, chemotherapy, and/or intervention therapy before surgery or treatment, and all of the patients accepted the detection of CTCs in peripheral blood before surgery. Patients with other systemic tumors and autoimmune diseases were excluded. This study was reviewed and approved by the hospital Ethics Committee (LW2019059), and all of the patients signed informed consent.

\section{Collection, processing, and tumor cell enrichment of peripheral blood samples}

EDTA anti-coagulation blood collection tubes were used to collect $5 \mathrm{ml}$ of peripheral blood samples from patients with liver cancer pre-operatively and mixed by inversion. Fifteen milliliters of red blood cell lysate were added to the peripheral blood samples, mixed well, and the cell pellet was resuspended with PBS. The residual cell pellet was fixed with formaldehyde at a final concentration of $4 \%$. The cells in the suspension were filtered using a CanPatrol ${ }^{\mathrm{TM}}$ system, and the fixed cells were transferred to a filter tube containing a filter, and the cells were filtered onto an $8 \mu \mathrm{M}$ filter using a vacuum pump. The filtered cell filter samples were fixed at room temperature using $4 \%$ formaldehyde.

\section{Multiple RNA in situ analysis}

The cells entrapped by the filter were typed using a multiplex RNA probe and the level of the cell CXCR4 gene expression was detected. According to the target gene type of the probe, specific capture probe epithelial biomarker probes (EpCAM and CK8/18/19), interstitial biomarker probes (vimentin and twist), and a leukocyte marker (CD45) were added for hybridization (see Table 2 for probe sequences).

First, the cells were pre-treated with a permeabilizing agent and a protease. Subsequently, three sets of RNA probes were added, incubated, and branched DNA (bDNA) signal amplification probes were added. Thus, 
Table 1 Baseline data of 99 patients enrolled in CTCs

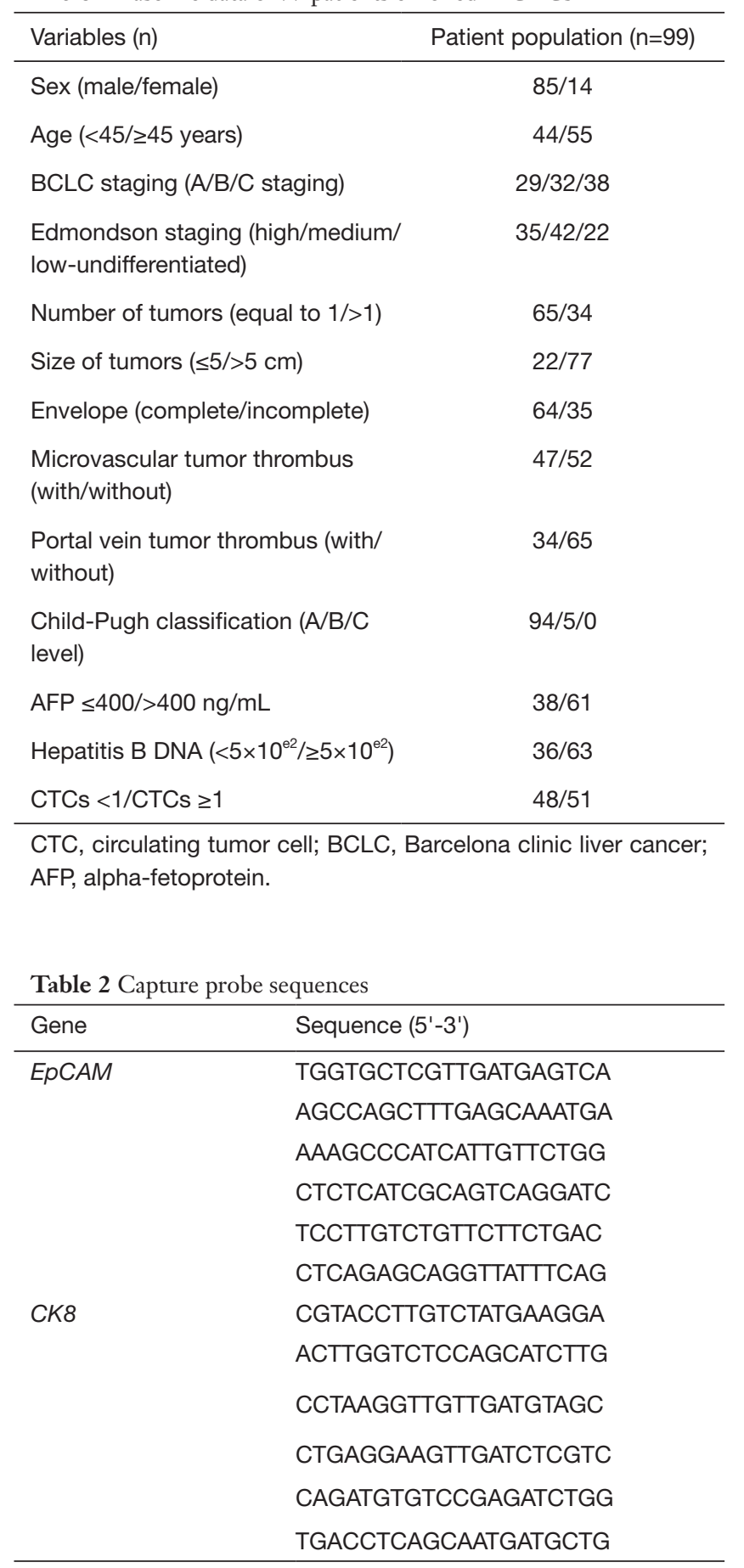

Table 2 (continued)
Table 2 (continued)

\begin{tabular}{|c|c|}
\hline Gene & Sequence $\left(5^{\prime}-3^{\prime}\right)$ \\
\hline \multirow[t]{6}{*}{ CK18 } & AGAAAGGACAGGACTCAGGC \\
\hline & GAGTGGTGAAGCTCATGCTG \\
\hline & TCAGGTCCTCGATGATCTTG \\
\hline & CAATCTGCAGAACGATGCGG \\
\hline & AAGTCATCAGCAGCAAGACG \\
\hline & CTGCAGTCGTGTGATATTGG \\
\hline \multirow[t]{6}{*}{ CK19 } & CTGTAGGAAGTCATGGCGAG \\
\hline & AAGTCATCTGCAGCCAGACG \\
\hline & CTGTTCCGTCTCAAACTTGG \\
\hline & TTCTTCTTCAGGTAGGCCAG \\
\hline & CTCAGCGTACTGATTTCCTC \\
\hline & GTGAACCAGGCTTCAGCATC \\
\hline \multirow[t]{6}{*}{ Vimentin } & GAGCGAGAGTGGCAGAGGAC \\
\hline & CTTTGTCGTTGGTTAGCTGG \\
\hline & CATATTGCTGACGTACGTCA \\
\hline & GAGCGCCCCTAAGTTTTTAA \\
\hline & AAGATTGCAGGGTGTTTTCG \\
\hline & GGCCAATAGTGTCTTGGTAG \\
\hline \multirow[t]{6}{*}{ Twist } & ACAATGACATCTAGGTCTCC \\
\hline & CTGGTAGAGGAAGTCGATGT \\
\hline & CAACTGTTCAGACTTCTATC \\
\hline & CCTCTTGAGAATGCATGCAT \\
\hline & TTTCAGTGGCTGATTGGCAC \\
\hline & TTACCATGGGTCCTCAATAA \\
\hline \multirow[t]{6}{*}{$C D 45$} & TCGCAATTCTTATGCGACTC \\
\hline & TGTCATGGAGACAGTCATGT \\
\hline & GTATTTCCAGCTTCAACTTC \\
\hline & CCATCAATATAGCTGGCATT \\
\hline & TTGTGCAGCAATGTATTTCC \\
\hline & TACTTGAACCATCAGGCATC \\
\hline \multirow[t]{8}{*}{ CXCR4 } & ACTGCCTTGCATAGGAAGTT \\
\hline & GGTTGACTGTGTAGATGACA \\
\hline & AGGATGAGGACACTGCTGTA \\
\hline & TAGCGGTCCAGACTGATGAA \\
\hline & CAACATAGACCACCTTTTCA \\
\hline & AATAGTCAGCAGGAGGGCAG \\
\hline & CGTTGGCAAAGATGAAGTCG \\
\hline & TATCTGTCATCTGССТСАСТ \\
\hline
\end{tabular}


Table 3 Sequences for the bDNA signal amplification probes

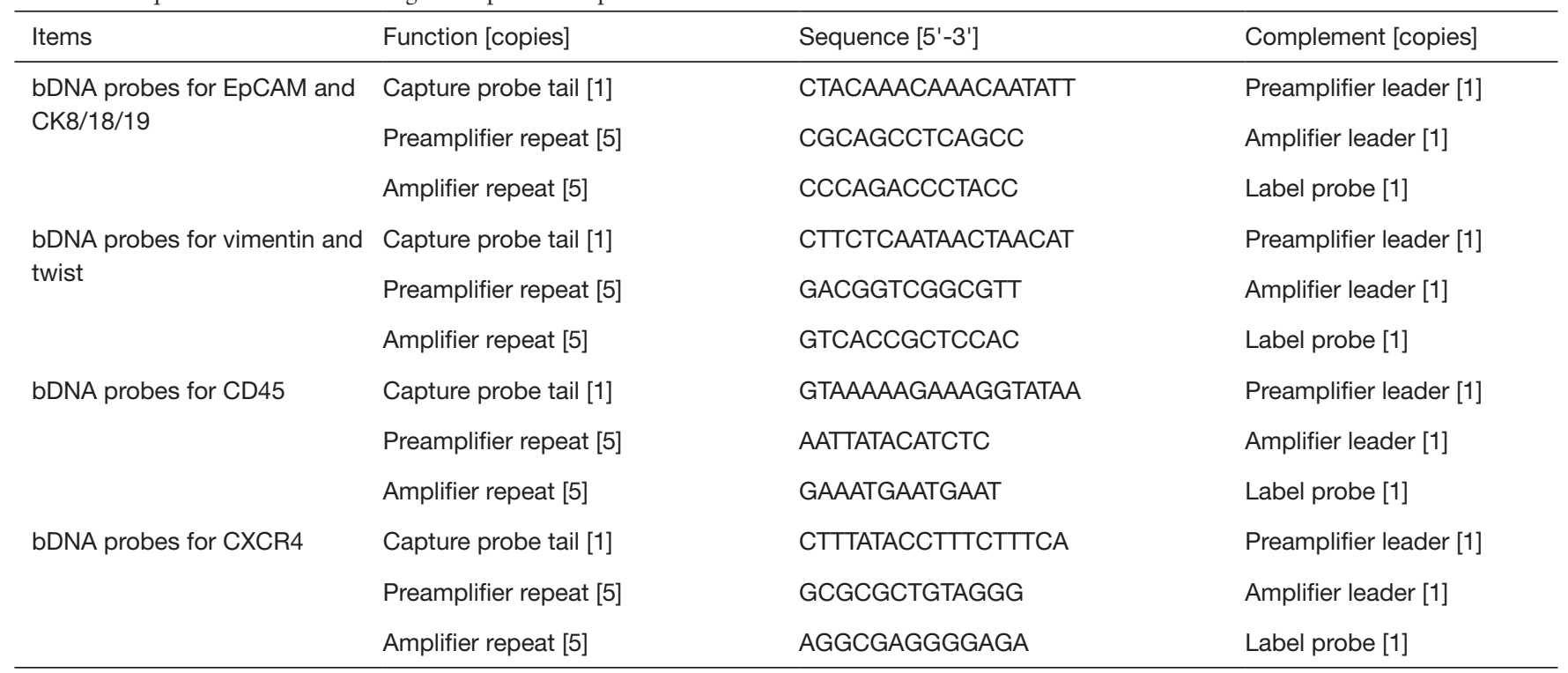

bDNA, branched DNA.

there were four labeled fluorescent proteins (see Table 3 for probe sequences), as follows: Alexa Fluor 594 [for labeled epithelial biomarker probes (EpCAM and CK8/18/19)]; Alexa Fluor 488 [interstitial biomarker probes (vimentin and twist)]; Alexa Fluor 750 [for labeling the leukocyte marker (CD45)]; and Alexa Fluor 647 (for labeling CXCR4) in combination with an RNA probe. Finally, the nuclei were stained with DAPI and observed under a fluorescence microscope (Olympus, Tokyo, Japan). The red and green fluorescent signal points represented the expression of epithelial and mesenchymal genes on CTCs, respectively. White signal dots represented the leukocyte marker (CD45) gene expression. Purple signal dots represented CXCR4 gene expression (see Figure 1).

\section{Clinical pathologic indicators}

Intrahepatic recurrent cancer and extrahepatic metastases were found within 2 years post-operatively on reexamination and considered to be early tumor recurrences. The diameter of the tumor refers to the maximum diameter of the tumor. MVI referred to tumor tissues connected to or filling the vascular lumen on observation with a microscope. The tumor capsule referred to a clear demarcation between tumor and surrounding tissues on observation with unaided eyes. The surrounding tissues were oppressed with tumor and a fibrous capsule. Non-enveloping refers to an unclear boundary between the tumor and the surrounding tissue. The Edmondson-Stein grade standard was used to evaluate the degree of differentiation of liver cancer. The Barcelona clinic liver cancer (BCLC) was used as the staging standard for liver cancer.

\section{Follow-up}

Patients were asked to undergo follow-up evaluations every month for 3 months, every 2 months for the following 6 months, and every 3 months for the next 6 months.

The following tests were obtained during followup evaluations: blood tests, including a routine blood examination, liver and kidney function tests, alphafetoprotein (AFP), and electrolytes; and imaging tests, including chest radiography, abdominal ultrasonography, and computed tomography/magnetic resonance imaging. Recurrence was defined as new HCC lesions present on computed tomography or magnetic resonance imaging with or without abnormal AFP levels. The primary outcome in our study was progression-free survival (PFS). The endpoint for disease-free survival (DFS) was HCC recurrence and death.

\section{Statistical analysis}

Statistical analysis of the data was performed using 


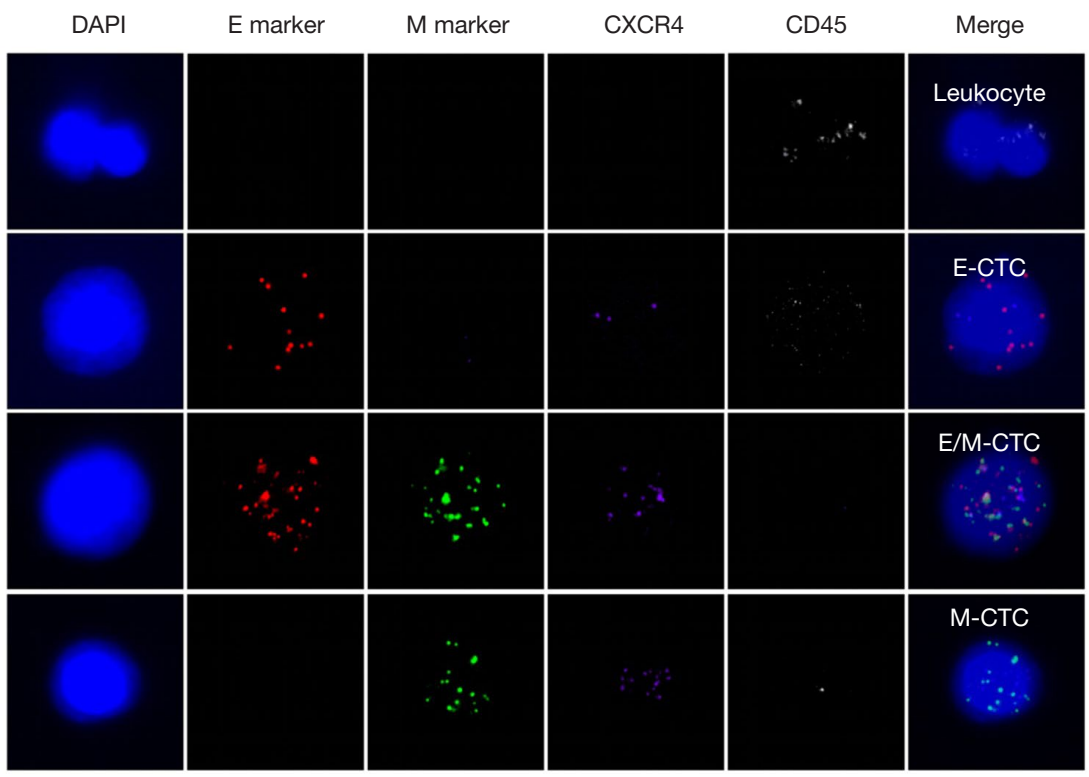

Figure 1 Detection and classification of CTCs using EMT markers. The red and green fluorescent signal points represented the expression of epithelial and mesenchymal genes on CTC, respectively. White signal dots represented the leukocyte marker (CD45) gene expression. Purple signal dots represented CXCR4 gene expression. CTC, circulating tumor cell; EMT, epithelial-mesenchymal transition.

SPSS22.0. Measurement data are expressed as the $\overline{\mathrm{x}} \pm \mathrm{S}$, and $t$-tests were used for the treatment of the data. A $\chi^{2}$ test was used to compare the difference in enumeration data and the difference was statistically significant at a $\mathrm{P}<0.05$. The Kaplan-Meier method was used to calculate the PFS.

\section{Results}

\section{Correlation analysis of CTCs and clinical pathologic indices}

The positive rate of CTCs was $89.9 \%$. The relationship between CTCs and various clinical indicators of liver cancer was based on the CTC-positive rate, number of CTCs, types of CTCs, and the proportion of interstitial CTCs. All of the CTCs detection results were analyzed from the pre-operative results, with a focus on the clinical correlation between CTCs and BCLC stage, Edmondson classification, Child-Pugh classification, tumor diameter, tumor number, capsule invasion, MVI, surgical margins, portal vein tumor thrombus, liver cirrhosis, and AFP, alanine transaminase (ALT) levels, hepatitis B DNA, and HBsAg levels. A significant correlation existed between BCLC stage, Edmondson classification, tumor diameter, tumor number, capsule invasion, portal vein tumor thrombus, hepatitis B DNA and the total number of CTCs and distribution of CTCs types, the correlations were positive and the Edmondson classification had a negative correlation $(\mathrm{P}<0.05)$. No correlation existed with MVI, surgical margins, liver cirrhosis, Child-Pugh classification, HBsAg levels, and ALT levels ( $\mathrm{P}>0.05)$, as shown in Table 4.

\section{Correlation between BCLC stage of liver cancer and number and types of CTCs}

Logistic regression analysis of all the BCLC patient data showed that there was a positive correlation $(\mathrm{P}=0.021)$. There was no correlation between epithelial CTCs and BCLC. There was a positive correlation between the number of mixed CTCs and stage ( $\mathrm{P}=0.033)$. There was a positive correlation between the number of interstitial CTCs and stage $(\mathrm{P}<0.001)$; shown in Figure 2.

\section{Correlation between CXCR4-positive expression and type of CTCs}

There was a significant difference between CXCR4 expression in various types of CTCs. The epithelial CTCs did not generally express CXCR4, while the mixed CTCs mostly expressed CXCR4. The number of interstitial CTCs 
Table 4 Correlation analysis between CTCs and every clinical liver cancer index

\begin{tabular}{|c|c|c|c|c|c|c|c|c|}
\hline Spearman's rho & $\mathrm{N}$ & Value & $\begin{array}{c}\text { CTCs positive or } \\
\text { not }\end{array}$ & $\begin{array}{l}\text { CTCs total } \\
\text { number }\end{array}$ & Epithelial & Mixed & Interstitial & $\begin{array}{c}\text { Interstitial CTCs } \\
\text { proportion }\end{array}$ \\
\hline BCLC stage $0-A / B / C$ & $\mathrm{~N}=99$ & $r$ & 0.248 & 0.221 & -0.032 & 0.265 & 0.419 & 0.407 \\
\hline Edmondson stage $\mathrm{I} / \mathrm{II} / \mathrm{III}$ & $\mathrm{N}=99$ & r & -0.252 & -0.374 & -0.443 & -0.271 & -0.235 & -0.097 \\
\hline $\begin{array}{l}\text { Tumor number (single/ } \\
\text { multiple) }\end{array}$ & & $P$ value & 0.917 & 0.132 & 0.71 & 0.236 & $<0.001^{\star \star}$ & $0.009^{\star \star}$ \\
\hline \multirow[t]{2}{*}{ Tumor diameter $(\leq 5 />5 \mathrm{~cm})$} & $\mathrm{N}=99$ & $r$ & 0.148 & 0.109 & -0.130 & 0.210 & 0.215 & 0.189 \\
\hline & & $P$ value & 0.158 & 0.300 & 0.217 & $0.045^{\star}$ & $0.040^{*}$ & 0.071 \\
\hline $\begin{array}{l}\text { Portal vein tumor thrombus } \\
\text { (no/yes) }\end{array}$ & & $P$ value & 0.251 & $0.018^{*}$ & 0.236 & $0.010^{*}$ & $0.006^{\star \star}$ & $0.007^{\star \star}$ \\
\hline \multirow[t]{2}{*}{ AFP (normal/abnormal) } & $\mathrm{N}=99$ & $P$ value & 0.083 & 0.193 & 0.224 & 0.192 & 0.206 & 0.132 \\
\hline & & $P$ value & 0.441 & 0.070 & $0.035^{*}$ & 0.071 & 0.053 & 0.218 \\
\hline \multirow[t]{2}{*}{ Hepatitis B-DNA (no/yes) } & $\mathrm{N}=99$ & r & 0.096 & 0.299 & 0.251 & 0.215 & 0.039 & -0.103 \\
\hline & & $P$ value & 0.379 & $0.005^{\star \star}$ & $0.020^{\star}$ & $0.046^{*}$ & 0.720 & 0.345 \\
\hline
\end{tabular}

A significant correlation was suggested when *, $\mathrm{P}<0.05$ (2-tailed) or ${ }^{*}, \mathrm{P}<0.01$ (2-tailed). CTC, circulating tumor cell; BCLC, Barcelona clinic liver cancer; AFP, alpha-fetoprotein.

expressing CXCR4 was similar to the number of interstitial CTCs not expressing CTCs, as shown in Table 5.

\section{Subsistence analysis}

A total of 99 patients underwent CTCs testing prior to surgery [see Table 6 for Comparison of baseline data of interstitial CTCs $<1$ and $\geq 1$ (classification data)], all of them had follow-up information related to disease recurrence and 72 had disease progression. We divided the CTCs into group A $(<5)$ and group B $(\geq 5)$, the epithelial and mixed CTCs into group A1 $(<3)$ and group B1 $(\geq 3)$, and the interstitial CTCs into group A2 $(<1)$ and group B2 $(\geq 1)$. Pre-operatively, there were 40 patients in group A and 59 in group B. The median PFS was 15.8 and 5.2 for groups $\mathrm{A}$ and $\mathrm{B}$, respectively. There was no significant difference in PFS between the two groups ( $\mathrm{P}=0.097)$. Pre-operatively, there were 63 patients in epithelial CTCs group A1 and 36 in group B1. The median PFS was 7.0 and 6.0 for group A1 and B1, respectively. There was no significant difference in
PFS between the two groups $(\mathrm{P}=0.692)$. Pre-operatively, there were 48 patients in interstitial CTCs group A2 and 51 patients in group B2. The median PFS was 13.3 and 5.0 for group A2 and group B2, respectively. Group A2 had a significantly longer PFS than group $\mathrm{B} 2(\mathrm{P}<0.05$; Figure 3$)$.

\section{Discussion}

Radical resection of HCC as a routine treatment has made the 5 -year survival rate of small and large HCC increase to $50-60 \%$ and $30-40 \%$, respectively, but a significant number of patients still die of liver cancer recurrence and metastasis after surgery (9). Tumor metastasis involves tumor cell shedding from the tumor, entering the bloodstream, and transfer to a distant place (10). CTCs represent various types of tumor cells present in the peripheral blood. CTCs that have left the primary lesion and entered the blood circulation (11) are considered to be important factors in the recurrence and metastasis of malignant tumors (12). After a thorough investigation, CTCs have been shown 

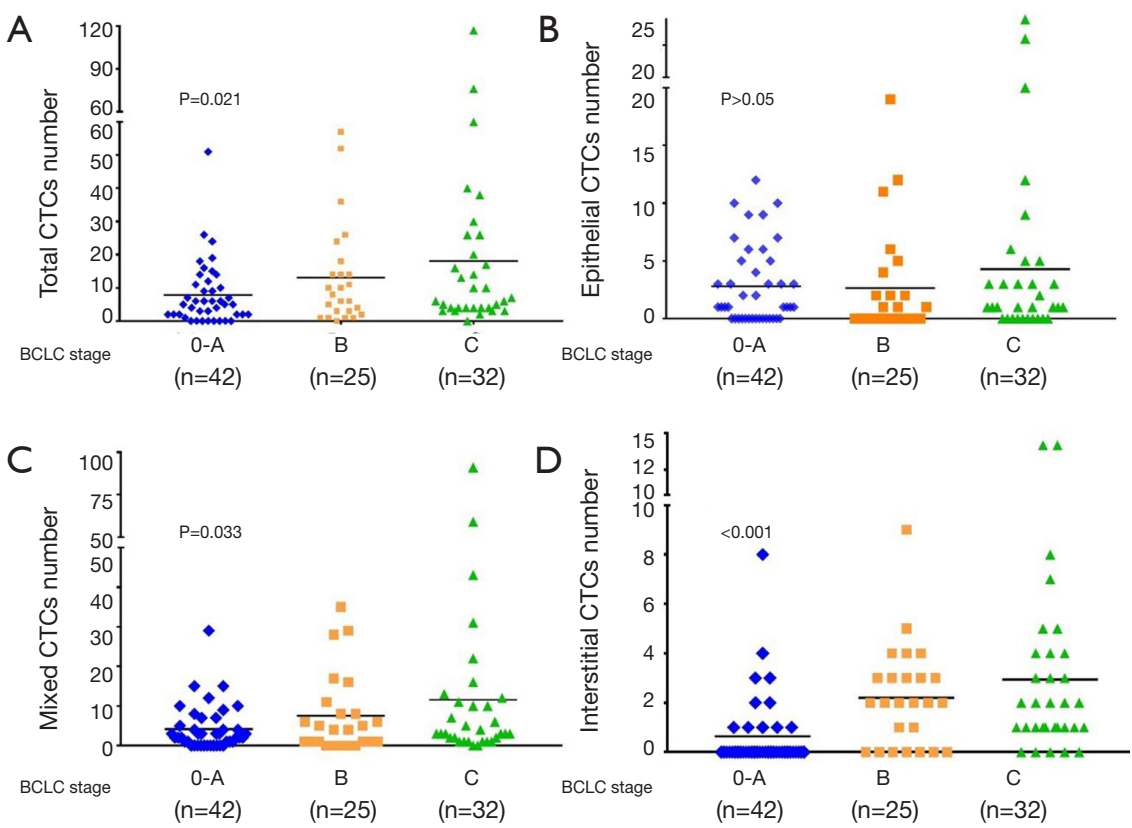

Figure 2 Correlation between BCLC stage of liver cancer and number and types of CTCs. (A) Liver cancer BCLC stage and CTCs total number; (B) liver cancer BCLC stage and epithelial CTCs number; (C) liver cancer BCLC stage and mixed CTCs number; (D) liver cancer BCLC stage and interstitial CTCs number. BCLC, Barcelona clinic liver cancer; CTC, circulating tumor cell.

Table 5 Correlation between CXCR4 positive expression and CTCs typing

\begin{tabular}{lccc}
\hline \multirow{2}{*}{ CTCs typing } & Cells number & \multicolumn{2}{c}{ CXCR4 expression } \\
\cline { 3 - 4 } & & $50(66.7)$ & Positive (\%) \\
\hline Epithelial & 75 & $63(30.6)$ & $25(33.3)$ \\
Mixed & 206 & $27(45.8)$ & $143(69.4)$ \\
Interstitial & 59 & $140(41.2)$ & $32(54.2)$ \\
Total & 340 & $200(58.8)$ & $<0.001$ \\
\hline
\end{tabular}

CTC, circulating tumor cell.

to have great significance in cancer diagnosis, treatment, evaluation of prognosis, and individualized treatment (13). Improved Can Patrol CTC enrichment technology has divided CTCsnto three subtypes according to EMT markers (epithelial, interstitial, and mixed types) (14). The results showed that more interstitial CTCs were detected in metastatic lesions. In a study of breast cancer (15), interstitial CTCs were predominant in blood samples from patients with aggressive breast cancer types. In the current study, CTCs were detected in 99 liver cancer patients preoperatively. CTCs are classified into different types. It was found that the positive rate of interstitial CTCs increased with the increase in BCLC stage. At the same time, interstitial CTCs and the positive rates of interstitial CTCs were the same as the total number of CTCs, which were significantly correlated with high-risk relapse indicators of HCC, such as tumor diameter, number, envelope, portal vein tumor thrombus, and AFP and hepatitis B DNA levels. This finding further indicated that CTCs that express EMT markers, i.e., interstitial and mixed CTCs, were greater in number and metastatic.

It has been shown that the positive rate of CTCs in peripheral blood of patients with liver cancer are positively correlated with the tumor stage (BCLC and TNM stage), and the positive rate of CTCs in patients with combined thrombus and extrahepatic metastasis is higher (16). Xu 

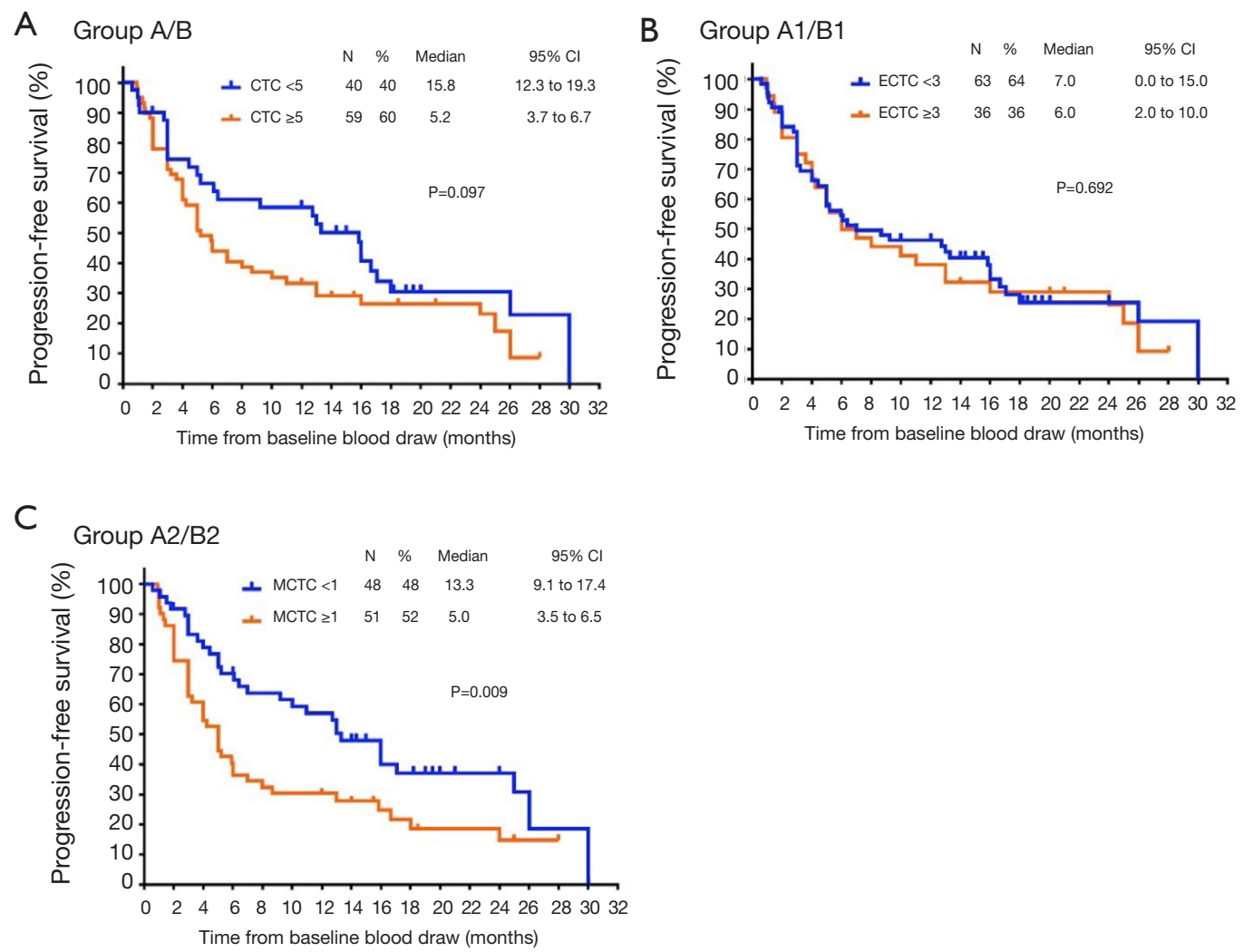

Figure 3 Preoperative CTCs number/type and PFS of patients. CTCs were divided into group A $(<5)$ and group B $(\geq 5)$, the epithelial and mixed CTCs into group A1 (<3) and group B1 $(\geq 3)$, and the interstitial CTCs into group A2 (<1) and group B2 ( $\geq 1)$. CTC, circulating tumor cell; PFS, progression-free survival.

et al. (17) reported 85 HCC patients with ASGPR and their ligands, and found that $80 \%$ with early or liver cancer $<2 \mathrm{~cm}$ had CTCs in the peripheral blood. Our findings were consistent with the $\mathrm{Xu}$ et al. study (17), further clarifying the role of CTCs in the guidance of follow-up individualized treatment for different stages of liver cancer. If CTCs can be used in routine examinations, it will be beneficial in the early diagnosis and treatment of HCC patients, thus prolonging the DFS and overall survival. Previous studies have suggested that portal vein tumor thrombi are the source of tumor metastases (18). There is a close relationship between CTCs and portal vein tumor thrombi. The positive rate of CTCs in peripheral blood in patients with portal vein tumor thrombi is significantly higher than patients without portal vein thrombosis $(19,20)$. Vona et al. (21) used Cell Search System (CSS) to detect CTCs in 44 patients with liver cancer, and the detection rate was $52 \%$. $\mathrm{Xu}$ et al. (17) used reverse transcription-PCR (RT-PCR) to detect CTCs in 85 HCC patients and reported that the detection rate of CTCs was significantly correlated with the existence of portal vein tumor thrombi. Our study was consistent with the above findings. We further found that interstitial CTCs and the types of CTCs that contain a high proportion of interstitial cells have a significant correlation with portal vein thrombosis, further indicating that among CTCs, interstitial CTCs have an important role in tumor chemotaxis and metastasis.

There is an abundance of research results involving the correlation between CTCs and AFP. Nel et al. (22) found that AFP mRNA is $75 \%$ sensitive and $71 \%$ specific by detecting EpCAM + CTC-specific gene expression. Schulze et al. (23) reported that the AFP levels of liver cancer patients with EpCAM + CTC patients were $>400 \mathrm{ng} / \mathrm{mL}$. Xu et al. (17) detected the expression of MACE-1, SSX1, and CPF11 mRNA in peripheral blood CTCs of 85 patients using RT-PCR, and found that $65.9 \%$ of liver cancer patients contained at least one of the above gene mRNA species, while the three CTC antigen gene 


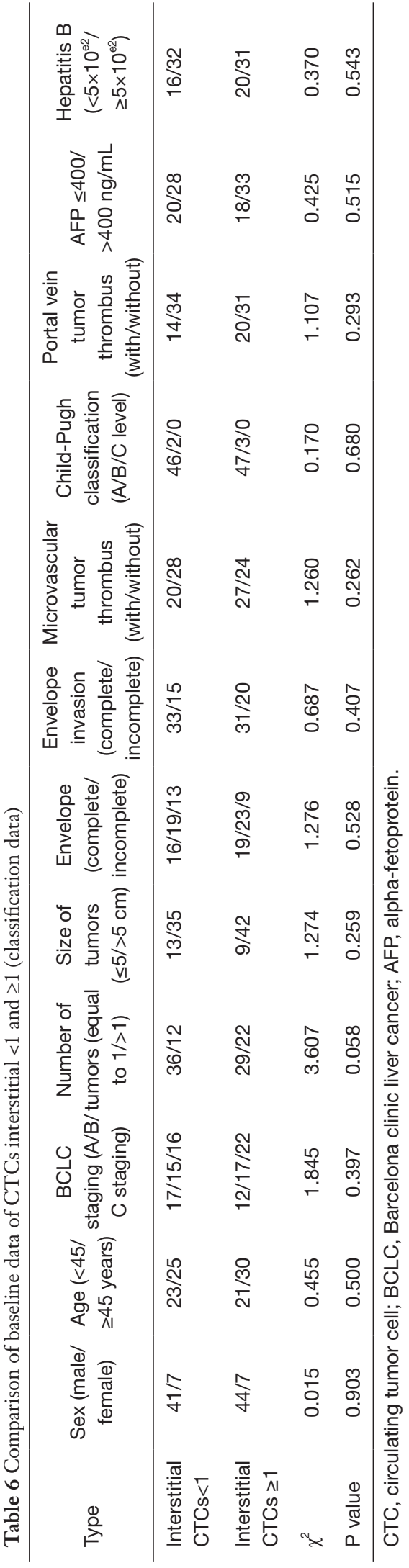

mRNA detection rate were not significantly correlated with serum AFP levels. Our study confirmed the correlation between epithelial CTCs and AFP, but did not fully prove the relationship between CTCs and AFP. Therefore, a large sample, multicenter study is needed to clarify the relationship between CTCs and AFP in patients with HCC.

CXCR4 and its receptor, CXCL12, has been shown to be closely related to the development and metastasis of various tumors, and the relationship between the high expression of the CXCR4 gene and CTCs has been found in a number of tumors (24). Mego et al. (25) showed that a relationship between breast cancer CTCs and CXCR4, and revealed the role of CXCR4-SDF-1 in the mobilization and transport of endothelial CTCs. However, in the HCC studies, the data did not match with other tumors. The expression of CXCR4 in tumors was with no significant difference (26) or even lower (27) compared with normal tissues by immunohistochemistry or PCR analysis. Recently, Kaemmerer et al. (28) and other studies found that CXCR4 was strongly expressed in the tumor blood vessels of liver cancer, and its CXCR4 expression was associated with poor prognosis. Yu et al. (29) showed that peripheral MMP26 + CXCR4 + were liver cancer CTCs, and considered that CXCR4 could be used as a marker for detecting CTCs. Our previous laboratory study confirmed that the "pathways in cancer" in gene expression profile analysis of HCCs with very high CTCs levels and those with very low CTCs levels revealed ranked first with respect to the differentially expressed genes. CXCR4 was shown to play an important part in seven cancer-related biological processes, some of which were metastasis-related biological process (e.g., positive regulation of cell invasion and migration, tumor angiogenesis, negative regulation of apoptosis, and chemotaxis) (30). We observed that most of the genes involved in these metastasis-related biological processes were up-regulated. Thus, some compensatory dissemination/metastasis mechanisms may exist in HCCs which may result in a high CTC count and M-CTCs percentage. Our study further found that the expression of CXCR4 in different types of CTCs was significantly different. The expression rate of CXCR4 in interstitial CTCs (interstitial and mixed CTCs) was $>50 \%$. This is in agreement with our previous laboratory findings.

To this end, we did tumor-free survival follow-up in 99 HCC patients who underwent CTCs before surgery, and found that with the exception of the number of preoperative CTCs, the types of CTCs all affected the tumorfree survival time. Although there is no statistical difference 
in the total number of CTCs, the trend was favorable. In terms of the total number of CTCs, 5 CTCs $/ 7.5 \mathrm{~mL}$ of peripheral blood were counted as the boundary value, and the tumor-free survival was significantly longer in patients with $<5$ CTCs compared to $>5$ CTCs. This affirmed the importance of CTCs as a post-operative prognostic indicator for liver cancer patients. The greater the expression of pre-operative peripheral blood CTCs, the poorer the prognosis. At the same time, in the research of different types of CTCs, we found that interstitial CTCs, with more invasion and metastatic potential, can be more accurate in the assessment of tumor-free survival time for liver cancer patients, which is consistent with other CTCs related to malignant tumors, metastatic breast cancer, colon cancer, and prostate cancer (31-33). Because of the small number of patients and limitation of the follow-up, only 22 patients had survival-related follow-up information, and total survival were not included in the study. Therefore, in future studies, large sample sizes and patient follow-up information will be refined for further validation.

In summary, peripheral CTC positivity is related to the recurrence and prognosis of $\mathrm{HCC}$ and can be used as an independent prognostic factor for HCC. At the same time, high CXCR4 protein expression was more common in mixed CTCs. The monitoring of peripheral blood CTCs should be a dynamic observation process combined with the characteristics of liver cancer to find the discipline of the changes in peripheral blood CTCs and guide clinical treatment. We bring new hope for accurately judging the clinical staging of liver cancer, guiding individualized treatment, and evaluating the prognosis.

\section{Acknowledgments}

Funding: This study was supported by Key Laboratory of Early Prevention and Treatment for Regional High Frequency Tumor, Ministry of Education (GK2018-01), National Natural Science Foundation Regional Fund Project (81860502), Key Project of Guangxi Science and Technology Department (Gui Ke AB16380242), Guangxi Colleges and University Young Teachers' Basic Ability Improvement Project (2017KY0115), and Guangxi Natural Foundation Youth Fund Project (2017GXNSFBA198234).

\section{Footnote}

Conflicts of Interest: All authors have completed the ICMJE uniform disclosure form (available at http://dx.doi. org/10.21037/tcr.2020.01.14). The authors have no conflicts of interest to declare.

Ethical Statement: The authors are accountable for all aspects of the work in ensuring that questions related to the accuracy or integrity of any part of the work are appropriately investigated and resolved. The study was conducted in accordance with the Declaration of Helsinki (as revised in 2013). This study was reviewed and approved by the hospital Ethics Committee (LW2019059), and all of the patients signed informed consent.

Open Access Statement: This is an Open Access article distributed in accordance with the Creative Commons Attribution-NonCommercial-NoDerivs 4.0 International License (CC BY-NC-ND 4.0), which permits the noncommercial replication and distribution of the article with the strict proviso that no changes or edits are made and the original work is properly cited (including links to both the formal publication through the relevant DOI and the license). See: https://creativecommons.org/licenses/by-nc-nd/4.0/.

\section{References}

1. Ferlay J, Soerjomataram I, Dikshit R, et al. Cancer incidence and mortality worldwide: sources, methods and major patterns in GLOBOCAN 2012. Int J Cancer 2015;136:E359-86.

2. Forner A, Reig M, Bruix J. Hepatocellular carcinoma. Lancet 2018;391:1301-14.

3. Díaz-González Á, Reig M, Bruix J. Treatment of hepatocellular carcinoma. Dig Dis 2016;34:597-602.

4. Ferreira MM, Ramani VC, Jeffrey SS. Circulating tumor cell technologies. Mol Oncol 2016;10:374-94.

5. Sundling KE, Lowe AC. Circulating tumor cells: overview and opportunities in cytology. Adv Anat Pathol 2019;26:56-63.

6. Kapeleris J, Kulasinghe A, Warkiani ME, et al. The prognostic role of circulating tumor cells (CTCs) in lung cancer. Front Oncol 2018;8:311.

7. Ding J, Wang K, Tang WJ, et al. Construction of epidermal growth factor receptor peptide magnetic nanovesicles with lipid bilayers for enhanced capture of liver cancer circulating tumor cells. Anal Chem 2016;88:8997-9003.

8. Salgia R, Weaver RW, McCleod M, et al. Prognostic and predictive value of circulating tumor cells and CXCR4 expression as biomarkers for a CXCR4 peptide antagonist in combination with carboplatin-etoposide in small cell lung cancer: exploratory analysis of a phase II study. Invest New Drugs 2017;35:334-44. 
9. Shi Z, Zhang $\mathrm{Y}$, Yin $\mathrm{Z}$. Therapeutic targeting of circulating tumor cells--a new strategy in treatment of liver cancer recurrence and metastasis after hepatectomy. Zhonghua Zhong Liu Za Zhi 2014;36:401-4.

10. Krzykawski MP, Krzykawska R, Paw M, et al. A novel in vitro model of metastasis supporting passive shedding hypothesis from murine pancreatic cancer Panc-02. Cytotechnology 2019;71:989-1002.

11. Dive C, Brady G. SnapShot: circulating tumor cells. Cell 2017;169:176.

12. Nel I, David P, Gerken GG, et al. Role of circulating tumor cells and cancer stem cells in hepatocellular carcinoma. Hepatol Int 2014;8:321-9.

13. de Wit S, van Dalum G, Terstappen LW. Detection of circulating tumor cells. Scientifica (Cairo) 2014;2014:819362.

14. Wu S, Liu S, Liu Z, et al. Classification of circulating tumor cells by epithelial-mesenchymal transition markers. PLoS One 2015;10:e0123976.

15. Yu M, Bardia A, Wittner BS, et al. Circulating breast tumor cells exhibit dynamic changes in epithelial and mesenchymal composition. Science 2013;339:580-4.

16. Zhao QW, Situ B, Zheng L. Current progress in research of circulating tumor cells. Nan Fang Yi Ke Da Xue Xue Bao 2017;37:1423-6.

17. $\mathrm{Xu} \mathrm{W}$, Cao $\mathrm{L}$, Chen $\mathrm{L}$, et al. Isolation of circulating tumor cells in patients with hepatocellular carcinoma using a novel cell separation strategy. Clin Cancer Res 2011;17:3783-93.

18. Jang TY, Huang CI, Yeh ML, et al. The prognosis of bulky hepatocellular carcinoma with nonmajor branch portal vein tumor thrombosis. Medicine (Baltimore) 2019;98:e15066.

19. Xia F, Lau WY, Xu Y, et al. Does hepatic ischemiareperfusion injury induced by hepatic pedicle clamping affect survival after partial hepatectomy for hepatocellular carcinoma? World J Surg 2013;37:192-201.

20. Sun YF, Xu Y, Yang XR, et al. Circulating stem cell-like epithelial cell adhesion molecule-positive tumor cells indicate poor prognosis of hepatocellular carcinoma after curative resection. Hepatology 2013;57:1458-68.

21. Vona G, Estepa L, Beroud C, et al. Impact of cytomorphological detection of circulating tumor cells in patients with liver cancer. Hepatology 2004;39:792-7.

22. Nel I, Baba HA, Weber F, et al. IGFBP1 in epithelial circulating tumor cells as a potential response marker to selective internal radiation therapy in hepatocellular carcinoma. Biomark Med 2014;8:687-98.

23. Schulze K, Gasch C, Staufer K, et al. Presence of EpCAMpositive circulating tumor cells as biomarker for systemic disease strongly correlates to survival in patients with hepatocellular carcinoma. Int J Cancer 2013;133:2165-71.

24. Zhu L, Zhang W, Wang J, et al. Evidence of CD90+CXCR4+ cells as circulating tumor stem cells in hepatocellular carcinoma. Tumour Biol 2015;36:5353-60.

25. Mego M, Cholujova D, Minarik G, et al. CXCR4-SDF-1 interaction potentially mediates trafficking of circulating tumor cells in primary breast cancer. BMC Cancer 2016;16:127.

26. Monnier J, Boissan M, L'Helgoualc'h A, et al. CXCR7 is up-regulated in human and murine hepatocellular carcinoma and is specifically expressed by endothelial cells. Eur J Cancer 2012;48:138-48.

27. Li N, Guo W, Shi J, et al. Expression of the chemokine receptor CXCR4 in human hepatocellular carcinoma and its role in portal vein tumor thrombus. J Exp Clin Cancer Res 2010;29:156.

28. Kaemmerer D, Schindler R, Mussbach F, et al. Somatostatin and CXCR4 chemokine receptor expression in hepatocellular and cholangiocellular carcinomas: tumor capillaries as promising targets. BMC Cancer 2017;17:896.

29. Yu C, Wang Z, Xu X, et al. Circulating hepatocellular carcinoma cells are characterized by CXCR4 and MMP26. Cell Physiol Biochem 2015;36:2393-402.

30. Qi LN, Xiang BD, Wu FX, et al. Circulating tumor cells undergoing EMT provide a metric for diagnosis and prognosis of patients with hepatocellular carcinoma. Cancer Res 2018;78:4731-44.

31. Cristofanilli M, Budd GT, Ellis MJ, et al. Circulating tumor cells, disease progression, and survival in metastatic breast cancer. N Engl J Med 2004;351:781-91.

32. Cohen SJ, Punt CJ, Iannotti N, et al. Relationship of circulating tumor cells to tumor response, progression-free survival, and overall survival in patients with metastatic colorectal cancer. J Clin Oncol 2008;26:3213-21.

33. Goldkorn A, Ely B, Quinn DI, et al. Circulating tumor cell counts are prognostic of overall survival in SWOG S0421: a phase III trial of docetaxel with or without atrasentan for metastatic castration-resistant prostate cancer. J Clin Oncol 2014;32:1136-42.

Cite this article as: Bai T, Mai R, Ye J, Chen J, Qi L, Tang J, Wei M, Zhang L, Chen Z, Tang Z, Li L, Wu F. Circulating tumor cells and CXCR4 in the prognosis of hepatocellular carcinoma. Transl Cancer Res 2020;9(3):1384-1394. doi: $10.21037 /$ tcr.2020.01.14 